\title{
Compromiso y gestión humana en la empresa*
}

Engagement and Human Management in the Enterprise

Recibido: 26 de septiembre de 2013 | Aceptado: 18 de marzo de 2016

\author{
Armando Cuesta Santos ** \\ Universidad Tecnológica de La Habana, Cuba
}

doi: 10.11144/ Javeriana.upsy15-2.cghe

Para citar este artículo: Cuesta Santos, A. (2016). Compromiso y gestión humana en la empresa. Universitas Psychologica, 15(2), 287-300. http://dx.doi. org/10.11144/Javeriana.upsy15-2.cghe

* Artículo de investigación científica y tecnológica.

** Profesor Titular de la Facultad de Ingeniería Industrial de la Universidad Tecnológica de La Habana (Instituto Superior Politécnico José Antonio Echeverría - Cujae). Presidente del Comité Académico y Coordinador de la Maestría en Gestión de Recursos Humanos de la Cujae. Consultor de Empresas. Facultad de Ingeniería Industrial. Correo electrónico: cuesta@ind.cujae.edu.cu

\section{RES UMEN}

En el ámbito de la gestión humana $(\mathrm{GH})$ la empresa, en el sentido de compromiso, es un constructo relevante. En el contexto de dicha gestión, su medición se constituye en un problema, en especial en su vínculo con el alto desempeño. Fue objetivo de la investigación realizada medir ese constructo, para insertarlo en el accionar de la GH en la empresa. Los instrumentos utilizados fueron la encuesta, la modelación matemática y la correlación estadística. Como resultados se obtuvieron modelos matemáticos para procesar la aplicación de instrumentos con sustentación teórica relativa al constructo estudiado, así como su validación para las peculiaridades socioculturales de los empleados de empresas cubanas, junto a su inserción conceptual en el accionar de la GH para su planificación y control de gestión estratégica. Palabras clave

gestión humana; compromiso; desempeño

\section{A B S T R A C T}

Engagement sense is a relevant construct in the human management at enterprise. The measurement of engagement sense in connection with the high performance at the HM context, is a problem. The measurement of this construct was the objective, in order to insert in the HM action. The survey, mathematic modeling and statistic correlation, were the methods utilized. As research results mathematics models for the instruments application were obtained, together the theory validation of the construct and their consideration for the social and cultural peculiarities of the Cuban enterprises employees, as well as their conceptual insertion in the HM action in order to their planning and strategic management control.

Keywords

human management; engagement; performance 


\section{Introducción}

El logro de un alto sentido de compromiso de los empleados con la organización es hoy motivo de preocupación en la empresa, considerando tanto el componente humanista al implicarse la persona socialmente como el económico al vincularse al aumento del desempeño (Beer, 2009; Boston Consulting Group \& World Federation of People Management Association, 2010; Boxall \& Macky, 2009; Drucker, 1999; Montoya \& Montoya, 2012; Picart, 2012; Schaufeli, Bakker, \& Van Rhenen, 2009).

En la encuesta mundial que reportaron en 2010 conjuntamente el Boston Consulting Group (BCG) y la World Federation of People Management Association (WFPMA), comprendiendo a un total de 109 países, se destacaron cuatro tópicos como los más críticos respecto a los recursos humanos: gestionar el talento, mejorar el desarrollo del liderazgo, realzar el compromiso de los empleados y planificar estratégicamente los recursos humanos (BGC \& WFPMA, 2010).

En la experiencia investigativa adquirida por el autor en el ámbito de la gestión humana $(\mathrm{GH})$ en la empresa, se han considerado activos intangibles como sentido de compromiso, competencias laborales, liderazgo, satisfacción, percepción de perspectivas (Cuesta, 2010; Cuesta \& Valencia, 2010). Entre los resultados del modelo de GH que el autor desarrolló en 2005, aplicado en empresas con fines de diagnosticar, planificar y controlar estratégicamente esa gestión (Cuesta, 2010, 2012), se destacaba el aumento del sentido de compromiso de los empleados.

Se ha constituido en un problema medir ese intangible en las empresas, con vistas a vincularlo con la planificación estratégica de la GH y el control de gestión estratégica. Este artículo tiene por objetivo reflejar los resultados de una investigación en empresas, referida a la medición del compromiso de los empleados, vinculándolo con el alto desempeño, con el fin de planificar el accionar de la GRH y concebir el control de gestión estratégico.

Resultan novedad científica los modelos matemáticos utilizados para procesar la aplicación de instrumentos con sustentación teórica relativa al constructo estudiado, así como la validación para las peculiaridades socioculturales de los empleados de empresas cubanas, junto a su inserción conceptual en el accionar de la GRH para su planificación y control de gestión estratégica.

\section{Método}

Las organizaciones laborales de dos empresas cubanas fueron el material objeto de estudio cuyos empleados tenían como peculiaridad sociocultural una alta calificación y un fuerte componente de investigación en sus resultados o productos finales. Los instrumentos utilizados fueron la encuesta, la modelación matemática y la correlación estadística.

Para medir "sentido de compromiso", se utilizaron tres instrumentos (encuestas) con el aporte de un modelo matemático a cada uno para su evaluación y contrastándolos mediante correlación estadística con el desempeño en dos estadios clasificatorios (bajo y alto) con el propósito de evidenciar la validez de criterio de modo concurrente y mediante expertos, así como la validez de constructo vinculado a la teoría que lo sustenta y sus correlaciones.

La conducción metodológica partió de reportes en la literatura científica de diversos autores que incluían al compromiso con el trabajo en sus estudios (sin perder la perspectiva de la búsqueda del accionar de la gestión humana en la empresa), implicando una teoría junto a instrumentos de evaluación, que, después en el desarrollo de la investigación, se asociaron a modelos matemáticos y se correlacionaron estadísticamente con el desempeño laboral (clasificado en alto y bajo desempeño laboral desde una perspectiva integral), discutiéndose los resultados empíricos alcanzados y posibilitando insertar ese constructo en la planificación estratégica de la empresa, así como en su control de gestión estratégica.

\section{Resultados}

La investigación bibliográfica condujo a un conjunto de autores actuales que venían estudiando el compromiso con el trabajo, en especial vinculado al alto desempeño y en el contexto particular de la GH 
en la empresa. En el modelo de Gestión de Recursos Humanos (GRH) desarrollado por Michael Beer y sus colaboradores del Harvard Businees School (Beer, Spector, Lawrence, Mills, \& Walton, 1985), se resaltan cuatro resultados fundamentales por alcanzar, siendo uno de ellos el compromiso de los empleados que conseguían el "alto compromiso":

Alto grado de compromiso: quiere decir que los empleados estarán motivados para oír, comprender y responder a las comunicaciones de la Gerencia respecto a los cambios en las demandas del entorno con sus implicaciones correspondientes en salarios, prácticas de trabajo y requerimientos de competencia. La mutua confianza contribuirá a que el mensaje de la Gerencia tenga mayor credibilidad para los empleados y a permitir que la Gerencia responda ante los legítimos intereses de los empleados como grupo de interés. ( p. 39)

En ese modelo, cuando definen compromiso ofreciendo a la vez una conducción metodológica que ha utilizado este autor (Cuesta, 2010) en la planificación de la $\mathrm{GH}-$, apuntan:

Compromiso: ¿Hasta qué punto sirven las políticas de GRH para aumentar el compromiso de las personas con su trabajo y con la organización? El refuerzo del compromiso puede tener por fruto no sólo un mayor grado de lealtad y un mayor rendimiento para la empresa, sino también el aumento de la autoestima, la dignidad, la implicación psicológica y la identidad de las personas. (Beer et al., 1985, p. 21)

Nótese de lo expresado sobre compromiso que no se limita solamente a la búsqueda del mayor rendimiento, sino que mediante la reciprocidad empleado-organización, indicada por la mutua confianza, se busca satisfacer intereses legítimos de los empleados, es decir, se considera además el aspecto del desarrollo humano, expresado en el aumento de la autoestima, de la dignidad y de la implicación psicológica. Se destaca la "motivación recíproca" en ese constructo pluridimensional que es el compromiso, lo cual se advierte como común en las teorías sobre compromiso que sustentan los tres instrumentos a los que se asocian los modelos matemáticos (Bakker \& Schaufeli, 2008; Cuesta, 2010; Eisenberger, Stinglhamer, Van Denberghe, Sucharski, \& Rhoades, 2002).

No obstante la teoría de Beer et al. (1985), a los efectos prácticos de medición del compromiso, no realizan propuestas de instrumentos. Las investigaciones de Michael Beer han continuado con esa orientación, destacando el nexo "alto compromiso-alto desempeño” (Beer, 2009). El citado modelo de Beer et al. (1985) fue referente del modelo de GH desarrollado por este autor (Cuesta, 2010, 2012) -donde el sentido de compromiso es el resultado más importante que se debe alcanzar por el accionar de la $\mathrm{GH}$ - que a su vez fue referente del modelo de $\mathrm{GH}$ para las empresas cubanas, refrendado para el proceso de perfeccionamiento empresarial en el país (Morales, 2006; Oficina Nacional Normalización, 2007). Sin embargo, ahí no se hacen propuestas de instrumentos.

Hay un conjunto reciente de investigaciones donde se resalta la importancia de alcanzar alto sentido de compromiso en los trabajadores, tanto por su implicación para el desarrollo humano como para el aumento del desempeño (Beer, 2009; BGC \& WFPMA, 2010; Chih-Hsun \& Hui, 2010; Montoya \& Montoya, 2012; Peralta, Santofimio, \& Segura, 2007; Raufflet, Lozano, Barrera, \& García, 2012; Ríos, Téllez, \& Ferrer, 2010), pero no aparece instrumentación para la evaluación.

Cada vez más es reconocida la tesis de que el desarrollo viene dado por la gente. En ello insiste el Programa de Desarrollo de las Naciones Unidas (PNUD) en su "Informe sobre Desarrollo Humano" que viene emitiendo desde 1990 (PNUD, 2010). Cada vez más el desarrollo o mejoramiento humano deviene sustento del mejoramiento empresarial sostenible.

La medición o valoración práctica de ese constructo es muy necesaria, y consecuentemente para este autor una consideración metodológica fundamental es la definición de medición, como constatación de práctica o empírea para develar el concepto, para su validación. Interesa esa constatación dado que la investigación aquí es para hacer gestión empresarial, para la transformación o el cambio en la práctica. Hay identificación con la siguiente definición de medición, concebida "como el proceso 
de vincular conceptos abstractos con indicadores empíricos" (Hernández, Fernández-Collado, \& Baptista, 2006, p. 276); quienes más adelante, respecto a la medición, en ese mismo sitio, apuntan:

La definición sugerida incluye dos consideraciones: la primera desde el punto de vista empírico y se resume en que el centro de atención es la respuesta observable $^{1}$ (sea una alternativa de respuesta marcada en un cuestionario, una conducta grabada vía observación o una respuesta dada a un entrevistador). La segunda es desde una perspectiva teórica y se refiere a que el interés se sitúa en el concepto $\underline{\text { subyacente no observable }}{ }^{2}$ que se representa por medio de la respuesta. (Hernández et al., 2006, p. 276)

1 Los subrayados son del autor de este artículo

2 Los subrayados son del autor de este artículo
Las consideraciones anteriores sobre metodología de investigación son además presupuestos básicos para el tratamiento de las referencias que siguen, incluyendo instrumentos aportados por autores que han desarrollado teorías en torno al constructo en cuestión.

Ese constructo de sentido de compromiso evidencia crecimiento o desarrollo humano, en tanto satisface la necesidad de pertenecer, de incluirse en una comunidad u organización -dado que la esencia humana la otorga el conjunto de relaciones sociales que integra la persona- $y$, a la vez, por esa pertenencia o compromiso, la persona trabajadora en reciprocidad busca, con su propio desempeño, cumplir el objetivo fundamental de la organización laboral, que es alcanzar mayores y mejores rendimientos. Esta última constatación fue sustento de la teoría del compromiso desarrollado por Eisenberger y sus colaboradores en 2002.

\section{TABLA 1}

Escala para la puntuación

Instrucción: En la encuesta siguiente hay ítems que representan posibles opiniones que Usted puede tener acerca de la organización donde está trabajando. Por favor indique el grado en que Usted está de acuerdo o en desacuerdo con cada cuestión o ítem, ubicando al lado el número del grado o puntuación que Usted considera según la escala siguiente:

\begin{tabular}{ccccccc}
\hline 0 & 1 & 2 & 3 & 4 & 5 & 6 \\
\hline $\begin{array}{c}\text { Fuertemente } \\
\text { en desacuerdo }\end{array}$ & $\begin{array}{c}\text { Moderadamente } \\
\text { en desacuerdo }\end{array}$ & $\begin{array}{c}\text { Ligeramente } \\
\text { en desacuerdo }\end{array}$ & $\begin{array}{c}\text { Ni en desacuerdo } \\
\text { ni en acuerdo }\end{array}$ & $\begin{array}{c}\text { Ligeramente } \\
\text { de acuerdo }\end{array}$ & $\begin{array}{c}\text { Moderadamente } \\
\text { de acuerdo }\end{array}$ & $\begin{array}{c}\text { Fuertemente } \\
\text { de acuerdo }\end{array}$ \\
\hline
\end{tabular}

Fuente: Rhoades y Eisenberger (2002).

TABLA 2

Encuesta de Percepción de Apoyo Organizacional (PAO)

\begin{tabular}{l}
\hline \multicolumn{1}{c}{ Ítem } \\
\hline 1. La organización valora mi contribución a su bienestar \\
2. La organización desestima cualquier esfuerzo extra de mi parte $(\mathrm{R}) *$ \\
3. La organización ignoraría cualquier descontento de mi parte $(\mathrm{R})$ \\
4. La organización realmente cuida de mi bienestar \\
5. Si hice bien el mejor trabajo posible, en la organización no se dan cuenta $(\mathrm{R})$ \\
6. La organización cuida de mi satisfacción general en el trabajo \\
7. La organización muestra poca preocupación por mí (R) \\
8. La organización se enorgullece de mis logros en el trabajo
\end{tabular}

*(R ). El contenido del ítem está planteado al contrario de la escala total.

Fuente: Rhoades y Eisenberger (2002). 
En 1986, se da a conocer la encuesta de Percepción de Apoyo Organizacional (PAO) desarrollada por Robert Eisenberger y colaboradores, que inicialmente constaba de 36 ítems y que con posterioridad se ha establecido con 8 ítems (Cherubin, 2011; Eisenberger et al., 2002; Rhoades \& Eisenberger, 2002). Se infería de esa percepción de apoyo a la persona por parte de la organización, un sentimiento de reciprocidad dado por el sentido de pertenencia o compromiso con esa misma organización, y un mejor desempeño o rendimiento por parte de la persona para satisfacerla. La encuesta de $\mathrm{PAO}$ precedida de la instrucción, donde se expresa la escala que se debe utilizar para la puntuación (Tabla 1), se presenta en la Tabla 2.

Robert Eisenberger junto con sus colaboradores y continuadores correlacionaron los distintos ítems (cada uno independientemente) con el sentido de pertenencia y reportaron que dichas correlaciones eran estadísticamente altas. Este autor, mediante un modelo matemático que revela la evaluación de la aplicación de ese instrumento con su conjunto de ítems, lo correlacionó con el desempeño, lo cual podrá apreciarse más adelante, mediante una propuesta de modelos matemáticos o formulaciones que comprende también a los dos instrumentos (encuestas) que seguirán. Otro estudio, de largo trayecto temporal, realizado por la Consultora Gallup (Gallup, 2010; Harter, Schmidt, Killham, \& Aspulud, 2006), ofrece también un instrumento evaluativo sobre el sentido de compromiso, pero por parte de este autor no se ha ejecutado labor de contraste empírico hasta el momento.

Se destaca otro instrumento de Eisenberger y colaboradores en esa misma dirección, pero no tratando el sentido de compromiso desde la inferencia que posibilita la $\mathrm{PAO}$, sino directamente, definiendo el sentido de compromiso mediante tres componentes: vigor, dedicación y absorción (Bakker \& Schaufeli, 2008; Schaufeli et al., 2009).

Ese instrumento, denominado Encuesta Utrecht de Escala de Compromiso con el Trabajo (UWES, por sus siglas en inglés), consta de 17 ítems y contempla tres componentes básicos que caracterizan, según los autores, el sentido de compromiso con el trabajo: vigor, dedicación y absorción. En la encuesta, seis ítems se refieren al vigor, que significa altos niveles de energía y resiliencia, completa ausencia de fatiga y persistencia ante los hechos y desafíos, representado por la frase: "en mi trabajo, yo siempre me siento con energía”. El segundo componente es la dedicación, con cinco ítems, se presenta cuando el trabajador otorga un sentido de importancia tal a su trabajo que lo impulsa a producir mejor, mostrando un sentimiento de inspiración y desafío, tal como se presenta en la siguiente afirmación: "yo encuentro en el trabajo un completo significado y propósito". Y el tercer componente es la absorción, con 6 ítems, se caracteriza por que el trabajador se encuentra totalmente inmerso y contento en su trabajo, superando dificultades y pasándole rápido el tiempo, siendo la frase que lo refleja: "cuando estoy trabajando, olvido cualquier otra cosa alrededor mío". La encuesta UWES también tiene siete rangos en su escala, pero en esta, respecto a la anterior, sí se manifiesta una linealidad o continuidad en su valoración (a diferencia de la encuesta de POA, se insiste), que va de 0 a 6 , indicando el valor 6 el máximo de sentido de compromiso.

En una investigación realizada por Cherubin (2011), se empleó la UWES de 17 ítems para evaluar la teoría del compromiso de la encuesta $\mathrm{PAO}$ de 8 ítems. Definió como variable independiente a la $\mathrm{PAO}$ y como variable dependiente al empleado comprometido, medido a través de la UWES. El estudio reveló, mediante correlación estadística, que cuando los empleados tienen la percepción del apoyo organizacional, el nivel de compromiso aumenta.

A continuación, se ofrece la instrucción que aportaron los autores, así como la encuesta ${ }^{3}$, reflejadas en la Tabla 3.

En el presente estudio, el autor desarrolló los dos siguientes modelos matemáticos o formulaciones, para reflejar mediante un indicador la evaluación

3 Aunque se perciben ajustados sus términos a la cultura de nuestros países latinoamericanos (se hizo pilotaje para verificar su inteligibilidad), solo se hicieron precisiones al añadir al ítem 15 el término "flexible" como similar a resiliente y en la instrucción ofrecida por los autores se especificó 1: mínimo y 6: máximo. 
TABLA 3

Encuesta Utrecht de Escala de Compromiso con el Trabajo (UWES)

Instrucción: Los 17 ítems siguientes tratan acerca de cómo Usted se siente en el trabajo. Por favor lea cada ítem cuidadosamente y decida si Usted se siente de ese modo en el trabajo. Si Usted nunca ha tenido ese sentimiento, marque $(\mathrm{X})$ en cero (0). Si Usted ha tenido ese sentimiento, indique en qué grado o nivel marcando el número (desde 1: mínimo, hasta 6: máximo).

\begin{tabular}{l}
$\begin{array}{l}\text { Ítem } \\
\text { 1. En mi trabajo, siempre estoy con mucha energía }\end{array}$ \\
\hline 2. Encuentro en el trabajo un completo significado y propósito \\
\hline 3. El tiempo vuela cuando estoy trabajando \\
\hline 4. En mi trabajo, me siento fuerte y vigoroso \\
\hline 5. Soy entusiasta respecto a mi trabajo \\
\hline 6. Cuando estoy trabajando, olvido lo que ocurre a mi alrededor \\
\hline 7. Mi trabajo me inspira \\
\hline 8. Al empezar el día siento que voy al trabajo \\
\hline 9. Me siento contento cuando estoy trabajando intensamente \\
10. Estoy satisfecho con el trabajo que realizo \\
11. Estoy inmerso en mi trabajo \\
12. Puedo continuar trabajando por largos periodos de tiempo \\
\hline 13. Para mí, el trabajo es un reto \\
14. Obtengo lo que quiero cuando estoy trabajando \\
15. En mi trabajo mentalmente soy muy resiliente (flexible) \\
16. La dificultad la separo de mí mismo en el trabajo \\
17. En mi trabajo siempre persevero cuando algo no va bien
\end{tabular}

Fuente: Schaufeli et al. (2009).

de cada una de esas encuestas, tanto de manera individual como colectiva.

Primero: Ipao $=\left[\left(\sum \mathrm{A} / 4\right)-\left(\sum \mathrm{R} / 4\right)\right] / 6$ (N), donde

Ipao: índice de percepción de apoyo organizacional.

$\sum$ A: sumatoria de las puntuaciones de cada uno de los ítems (4) en Anverso (A) -de

frente o lineal con la escala, in crescendo de $0 \mathrm{a}$ 6 muy positivo.

$\sum \mathrm{R}$ : sumatoria de las puntuaciones de cada uno de los ítems (4) denominados $\mathrm{R}$, en

Reverso $(\mathrm{R})$-al revés de la escala, in crescendo de 6 a 0 muy positivo.

$\mathrm{N}$ : cantidad total de empleados encuestados.

El índice Ipao oscila en valores continuos entre $-1,0$ y +1 . Un Ipao $=1$ significa el máximo de percepción de apoyo organizacional, y es lo que se pretende como muy positivo. Un Ipao $=-1$ significa el mínimo de percepción de apoyo organizacional (o inexistencia de esa percepción), y es lo que se evita por ser muy negativo.

Segundo: el indicador modelado de la Encuesta Utrecht de Escala de Compromiso con el Trabajo:

Inwes $=\left(\sum \mathrm{P} / 17\right) / 6(\mathrm{~N})$, donde

Iuwes: índice de compromiso con el trabajo atendiendo a la aplicación de la encuesta

Utrecht (UWES).

$\sum \mathrm{P}$ : sumatoria de las puntuaciones recibidas en cada uno de los 17 ítems de la

encuesta Utrecht.

$\mathrm{N}$ : cantidad total de empleados encuestados.

El valor que puede asumir Iuwes oscila entre 0 y 1 . La tendencia a 1 es lo que se pretende, porque significa sentido de compromiso con el trabajo; y la tendencia a cero (0) significa la pérdida o no tenencia de ese sentido de compromiso con el trabajo. 
Más adelante podrá apreciarse el rango clasificatorio que contextualiza o matiza necesariamente lo antes planteado. Y comprendiendo también teoría y metodología, este autor ha venido evaluando el Índice de sentido de compromiso (Isc) desde el modelo de GH concebido en 2005. En el Isc, se consideraron cinco subíndices, atributos o dimensiones, tomando como referente la argumentación sobre Alto grado de compromiso expuesta por Michael Beer y colaboradores (Beer et al., 1985). A continuación, se presentan la instrucción y la encuesta, con las cinco dimensiones o subíndices, comprendiendo cada una la escala de cinco rangos planteada.

Instrucción: Marque X en el rango $(1-2,3-4, \ldots$ 9-10) comprendido en cada una de las cinco dimensiones siguientes, donde Usted se ubica percibiendo su sentido de compromiso con su institución o empresa.

1. Identificación con la misión y valores de la institución

\begin{tabular}{|c|c|c|c|}
\hline 2 & 4 & 5 & 7 \\
\hline $\begin{array}{c}\text { Ninguna } \\
\text { e }(-1)\end{array}$ & $\begin{array}{c}\text { Poca } \\
\mathrm{d}(-0.5)\end{array}$ & $\begin{array}{l}\text { Media } \\
\text { c (0) }\end{array}$ & $\begin{array}{l}\text { Buena } \\
\text { b }(0.5)\end{array}$ \\
\hline
\end{tabular}

2. Fidelidad con la alta dirección de la institución:

3. Motivación para oír, comprender y responder a las comunicaciones de la gerencia respecto a los cambios en las demandas del entorno con sus implicaciones en salario, organización del trabajo, requisitos de competencias, etc.:

4. Disposición al trabajo:

5. Credibilidad en las aspiraciones esenciales de la institución y sus líderes:

Y a esa ponderación y lógica se corresponde el siguiente modelo:

\section{TABLA 4}

Contraste de la PAO con el Desempeño de los 65 encuestados $\sum \mathrm{a}(1)+\sum \mathrm{b}(0.5)+\sum \mathrm{c}(0)+\sum \mathrm{d}(-0.5)+\sum \mathrm{e}(-1)$

Isc

$\mathrm{N}$

donde,

Isc: índice de sentido de compromiso del empleado (i) o del grupo (j).

e, d, c, b, a: variables de probables marcajes $(X)$, significando ninguna, poca, media, buena y mucha, respectivamente.

$\mathrm{N}$ : cantidad de encuestados.

Seguidamente se muestran los resultados de la investigación de campo o empírica realizada con los instrumentos y sus correspondientes modelos matemáticos que los reflejan, explicados antes.

La aplicación de la encuesta de Percepción de Apoyo Organizacional (PAO) se hizo sobre una muestra representativa tomada del $90 \%$ de los trabajadores de las áreas productivas fundamentales de los dos casos empresariales del estudio, dando un total de 65 trabajadores $(\mathrm{N}=65)$, con el fin de relacionar el índice $\mathrm{PAO}$ con el nivel de desempeño. Cada encuestado se clasificó en una escala nominal, según dos estados: Alto Desempeño (AD) o Bajo Desempeño (BD) y respecto a la $\mathrm{PAO}$, en tres alternativas: Alta PAO, Baja PAO y Ninguna PAO, dando lugar a los resultados de la Tabla 4.

Atendiendo a los valores que puede asumir el modelo concebido para reflejar la $\mathrm{PAO}$, a saber,

Ipao $=\left[\left(\sum \mathrm{A} / 4\right)-\left(\sum R / 4\right)\right] / 6(\mathrm{~N})$, las tres alternativas de clasificación asumidas fueron las siguientes: Alta PAO: 0.5 a 1; Mediana PAO: 0.01 a

\begin{tabular}{ccccc}
\hline & Alto Desempeño (AD) & Bajo Desempeño (BD) & Total \\
\hline Alta PAO & 29 & 2 & 31 \\
\hline Mediana PAO & 6 & 8 & 14 \\
\hline Baja PAO & 4 & 16 & 20 \\
\hline Total & 39 & 26 & 65 \\
\hline
\end{tabular}

Fuente: elaboración propia. 
0.49. Ninguna Baja PAO: - 1 a 0. Se ilustra seguidamente cómo se determinó el Ipao de los encuestados $(1,2, \ldots 66)$ para luego clasificarlos: Ipao1 $=[(24 / 4)$ $-(0 / 4)] / 6(1)=1 ;$ Ipao $2=[(16 / 4)-(4 / 4)] / 6(1)$ $=0.5$; Ipao $3=[(1 / 4)-(23 / 4)] / 6(1)=-0.91 \ldots$ hasta Ipao 65, dado que $\mathrm{N}=65$.

La validez del instrumento se calculó mediante varias evidencias, en particular acudiendo a la validez concurrente y validez de expertos (Hernández et al., 2006). El criterio de validez del desempeño evaluado se obtuvo de la evaluación integral del desempeño anual junto al juicio de los respectivos jefes, pues se decidió como más abarcadora la valoración integral (considerando las responsabilidades, además del rendimiento) y no otro indicador sumario como productividad del trabajo o aprovechamiento de la jornada, que dejarían fuera otros atributos del desempeño, en particular las responsabilidades. Además de su correlación con el desempeño, se buscó la validez concurrente de la medición de la $\mathrm{PAO}$ con criterios de expertos (sus jefes) con base en el resultado de la encuesta.

Para percibir la asociación o correlación estadística entre $\mathrm{PAO}$ y desempeño se tuvieron en cuenta las limitaciones de la escala nominal, que vienen dadas por ser la de más bajo rango de medición, ya que solo nombra o nomina, no establece rangos (de menor a mayor), no hay un cero $(0)$ real y su distribución de frecuencias es desconocida, por lo que a los efectos de la inferencia estadística se descartan los estadígrafos paramétricos y algunos no paramétricos más potentes que al menos exigen medición ordinal. Se recurrió al estadígrafo no paramétrico Coeficiente de Contingencia C (Siegel, 1974), que es una medida del grado de asociación o relación entre dos conjuntos de atributos, singularmente útil cuando se tiene solamente información clasificatoria (escala nominal) acerca de uno o ambos conjuntos de atributos.

El grado de asociación entre dos conjuntos de atributos (Percepción de Apoyo Organizacional [PAO] y Desempeño Alto o Bajo), independientemente de la distribución de la población, puede observarse en una tabla de contingencia de las frecuencias (Tabla 5), mediante:

$$
C=\sqrt{\frac{\chi^{2}}{N+\chi^{2}}}
$$

donde, $\mathrm{r} \quad \mathrm{k}$

$$
\chi^{2}=\Sigma \Sigma(\mathrm{O} \text { ij }-\mathrm{Eij})^{2}
$$

(probándose la Ho mediante esta expresión de $\left.\chi^{2}\right)$

y donde,

$$
I=1 \quad j=1 \quad \text { Eij }
$$

Oij: es el número observado de casos clasificados en la fila i de la columna $j$.

Eij: es el número de casos esperados conforme a Ho que clasificarán en la fila i de la columna j.

$\Sigma \quad \Sigma$ : indica sumar en todas las filas (r) y en todas las columnas $(\mathrm{k})$, es decir, sumar en todas las celdillas.

A medida que la discrepancia entre los valores esperados y los valores observados de las celdillas es mayor, se incrementa el grado de asociación entre las dos variables y, por lo tanto, es mayor el valor de C.

Los valores de $\chi^{2}$ dados por la formula de $\mathrm{C}$ son distribuidos aproximadamente como chi cuadrada con $g l=(\mathrm{r}-1)(\mathrm{k}-1)$, donde $\mathrm{r}$ es el número de filas y $\mathrm{k}$ es el número de columnas en la tabla de contingencia. En la Tabla 5, la frecuencia esperada para la

\section{TABLA 5}

Frecuencias observadas y esperadas de PAO y Desempeño

\begin{tabular}{ccccccc}
\hline & Alto Desempeño (AD) & \multicolumn{2}{c}{ Bajo Desempeño (BD) } & Total \\
\hline Alta PAO & $(18.6)$ & 29 & $(12.4)$ & 2 & 31 \\
\hline Mediana PAO & $(8.4)$ & 6 & $(5.6)$ & 8 & 14 \\
\hline Baja PAO & $(12)$ & 4 & $(8)$ & 16 & 20 \\
\hline Total & & 39 & & 26 & 65 \\
\hline
\end{tabular}

Fuente: elaboración propia. 
celdilla inferior derecha es E32 = (26) (20) $/ 65=$ 8. Sustituyendo los valores obtenidos en la Tabla 5 en la expresión de $\chi^{2}$, se obtiene: $(29-18.6)^{2} / 18.6$ $+(2-12.4)^{2} / 12.4+(6-8.4)^{2} / 8.4+(8-5.6)$

$2 / 5.6+(4-12)^{2} / 12+(16-8)^{2} / 8=29.56$

Sustituyendo en el modelo que refleja C, se:

$$
C=\sqrt{\frac{29.56}{65+29.56}}=0.559
$$

Se acude a la tabla $\mathrm{C}$ de valores críticos de chi cuadrada (Siegel, 1974, p. 283), mediante $g l=2$ contrastándose que $\chi^{2}$ obtenida (29.56) $>\chi^{2}$ teórica (13.82). Por tanto, se rechaza Ho (que no existe correlación entre $\mathrm{PAO}$ y Desempeño) para $\alpha=0.001$.

La aplicación de la Encuesta Utrecht de Escala de Compromiso con el Trabajo (UWES) se hizo a una muestra representativa de 56 trabajadores $(\mathrm{N}$ = 56) (tomada del $90 \%$ de los trabajadores de las áreas productivas fundamentales de los dos casos empresariales del estudio), para relacionar el Iuwes con el nivel de Desempeño. Cada encuestado se clasificó (escala nominal) en dos estados: de Alto Desempeño (AD) o de Bajo Desempeño (BD) y, respecto a luwes, en tres alternativas: Alto, Mediano y Bajo compromiso. Se acudió igualmente al Coeficiente de Contingencia C, y los argumentos para ello fueron los antes referidos y el procedimiento, el mismo. Los resultados se muestran en la Tabla 6.

Atendiendo a los valores que puede asumir el modelo concebido para reflejar el Índice UWES (luwes), a saber: Iuwes $=\left(\sum P / 17\right) / 6(N)$, entonces las tres alternativas de clasificación asumidas fueron las siguientes: Alto sentido de compromiso: $0.5 \mathrm{a}$ 1; Mediano sentido de compromiso: 0.25 a 0.49 ; Bajo sentido de compromiso: 0 a 0.24 . Se ilustra seguidamente cómo se determinó el Iuwes de los encuestados $(1,2, \ldots$ 56) y luego la clasificación de cada encuestado en una de las tres alternativas (Tabla 6): Iuwes $1=(4 * 17 / 17) / 6(1)=4 / 6=0.66$; Iuwes $2=(6 * 17 / 17) / 6(1)=6 / 6=1$; Iuwes $3=$ $(1 / 17) / 6(1)=0.059 / 6=0.01 \ldots$ hasta luwes 56 , dado que $N=56$.

Sustituyendo los valores obtenidos en la Tabla 6, en la expresión de $\chi^{2}$ y en $\mathrm{C}$, se alcanzaron los valores de 16.57 y 0.477 , respectivamente. Con base en la tabla $C$ de valores críticos de chi cuadrado (Siegel, 1974, p. 283), mediante $g l=2$, se encuentra que $\chi^{2}$ obtenido (16.57) $>\chi^{2}$ teórico (13.82). Por tanto, se rechaza Ho (que no existe correlación entre Iuwes y Desempeño) para $\alpha=0.001$. La validez del instrumento se buscó con el mismo modus operandi utilizado para el instrumento anterior.

Por último, se trabajó en la búsqueda del aval empírico de la encuesta del Índice de sentido de compromiso (Isc) de cinco dimensiones. También se decidió validarlo por criterios similares. No se redundará en los argumentos del estadígrafo utilizado y el procedimiento, dado que fueron los mismos. En este caso, se aplicó la encuesta a los mismos trabajadores de la empresa en que se aplicó la encuesta del Ipao, aunque no se recibió la respuesta de 11 encuestados (ya que no se encontraban laborando en la empresa en esos momentos), pudiéndose procesar los resultados de $54(\mathrm{~N}=54)$. Los datos obtenidos se presentan en la Tabla 7. Al recurrir a su modelo, a saber:

$$
\begin{aligned}
\mathrm{Isc} & =\left[\sum \mathrm{a}(1)+\sum \mathrm{b}(0.5)+\sum \mathrm{c}(0)+\sum \mathrm{d}\right. \\
(-0.5) & \left.+\sum \mathrm{e}(-1)\right] / \mathrm{N}
\end{aligned}
$$

Las tres alternativas de clasificación asumidas fueron las siguientes: Alto sentido de compromiso: 2.5 a 5; Mediano sentido de compromiso: 0 a 2.49; Bajo sentido de compromiso: -5 a $-0,01$. Se ilustra

\section{TABLA 6}

Frecuencias observadas y esperadas de Iuwes y Desempeño

\begin{tabular}{lccccc}
\hline & Alto Desempeño (AD) & Bajo Desempeño (BD) & Total \\
\hline Alto sentido de compromiso & $(15.8)$ & 22 & $(10.2)$ & 4 & 26 \\
\hline Mediano sentido de compromiso & $(9.1)$ & 9 & $(5.9)$ & 6 & 15 \\
\hline Bajo sentido de compromiso & $(9.1)$ & 3 & $(5.9)$ & 12 & 15 \\
\hline Total & & 34 & & 22 & 56 \\
\hline
\end{tabular}

Fuente: elaboración propia. 
TABLA 7

Frecuencias observadas y esperadas de Isc y Desempeño

\begin{tabular}{|c|c|c|c|}
\hline & Alto Desempeño (AD) & Bajo Desempeño (BD) & Total \\
\hline Alto sentido de compromiso & $(14.8) \quad 23$ & $(10.1) \quad 2$ & 25 \\
\hline Mediano sentido de compromiso & $(7.7)$ & $(5.3)$ & 13 \\
\hline Bajo sentido de compromiso & $(9.4)$ & $(6.5)$ & 16 \\
\hline Total & 32 & 22 & 54 \\
\hline
\end{tabular}

Fuente: elaboración propia.

seguidamente cómo se determinó el luwes de los encuestados $(1,2, \ldots 56)$ para luego clasificar a cada encuestado en una de las tres alternativas siguientes (Tabla 7): Isc $1=125(1)+50(0.5)+25(0)+$ $25(-0.5)+25(-1) / 54=2.08 ;$ Isc $2=125(1)+$ $115(0.5)+10(0)+20(0.5)+0(-1) / 54=3.19$; Isc $3=30(1)+70(0.5)+0(0)+20(-0.5)+$ $150(-1) / 54=-1.75 \ldots$ hasta Isc 56 , pues $N=56$.

Al determinar $\chi^{2}$ se alcanzó un valor de 15.82 y una $\mathrm{C}=0.476$. Se acudió a la tabla $\mathrm{C}$ ya referida, mediante $g l=2$ contrastándose que $\chi^{2}$ obtenida (15.82) > $\chi^{2}$ teórica (13.82). Por tanto, se rechazó Ho (que no existe correlación entre Isc y Desempeño) para $\alpha=0.001$.

\section{Discusión}

Es necesario señalar que esos instrumentos fueron escogidos para establecer mediciones "antes-después" del accionar de la GH. A diferencia de los dispositivos para la medición de tangibles, como el termómetro de ${ }^{\circ} \mathrm{C}$ o la cinta métrica, que implican universalidad, el caso de los instrumentos para medir intangibles por lo general se consideran casuísticos y en esa relación "antes y después" respecto al mismo objeto de medición y en el escenario estratégico especifico. Como es sabido, podemos utilizar distintas encuestas de satisfacción laboral que si bien miden esa variable, están conformadas por distintos ítems y escalas, lo que impide que se utilicen indistintamente para diferentes empresas.

Para la gestión empresarial en general y la $\mathrm{GH}$ en particular, el indicador sentido de compromiso de los empleados con la organización, tiene una gran relevancia práctica, dado su nexo con el alto desempeño -como se pudo constatar en esta investiga- ción-y, en consecuencia, con la alta productividad y la utilidad o ganancia empresarial. Además de ese significado económico, su relevancia práctica se revela en el desarrollo humano al implicar la pertenencia o inclusión en una comunidad o conjunto de relaciones humanas, reforzando la autoestima y la dignidad, incluido el crecimiento humano o psicológico. Interesa entonces considerar y evaluar ese sentido de compromiso de los empleados, por su implicación en el aumento económico y el crecimiento o desarrollo humano. Se reconoce cada vez más que el desarrollo viene dado por la gente, por lo tanto, la sustentabilidad del crecimiento económico de la empresa vendrá dada mayormente por los alcances del desarrollo humano.

Los modelos matemáticos utilizados para la medición del sentido de compromiso han dado lugar a respectivos indicadores que facilitan los contrastes en la práctica empresarial con el alto y bajo desempeño (p. ej., en el caso que nos ocupa, a través de la correlación estadística mediante el Coeficiente de Concordancia C), infiriendo que a un alto sentido de compromiso corresponden también altos desempeños y viceversa. Esos indicadores pueden planificarse, más aún, en la actualidad deben incluirse en la planificación y en el control estratégico de gestión como activos intangibles de la empresa. La relación del indicador intangible, que es el sentido de compromiso, con el tangible, que es el desempeño laboral en su más plena dimensión (al comprender no solo productividad o aprovechamiento de la jornada, sino responsabilidades), tiene significado metodológico para la constatación empírica al incluir la correlación estadística.

Como se refirió sintéticamente en el marco teórico, el sentido de compromiso, como intangible 


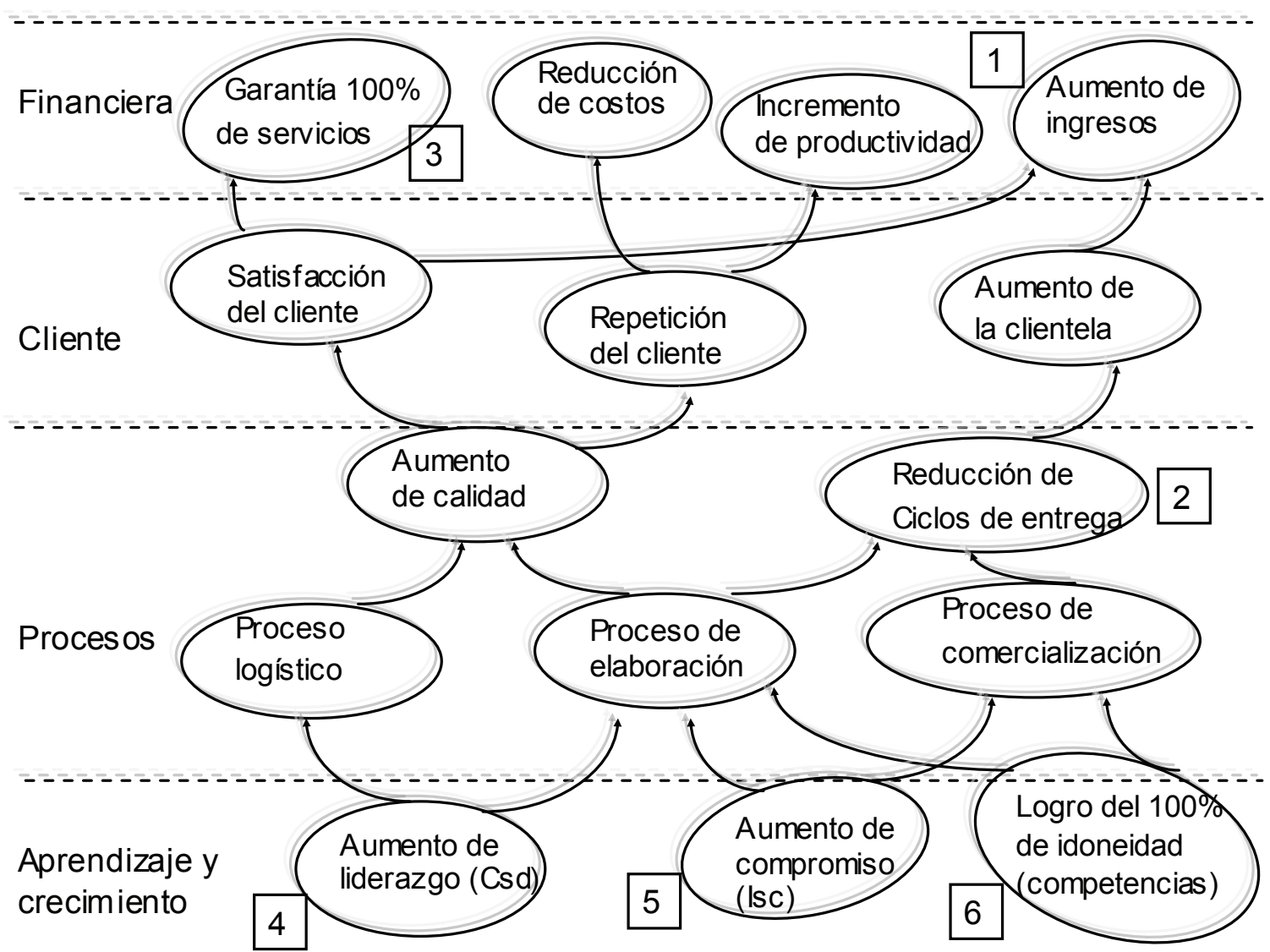

Figura 1. CMI para una empresa destacando los objetivos estratégicos en sus nexos causales.

Fuente: elaboración propia.

estratégico, está estrechamente ligado al desarrollo humano de la empresa, en tanto desarrollo de autoestima, dignidad, implicación social y crecimiento humano, ya que hoy es ampliamente aceptado que el crecimiento económico sustentable, depende esencialmente del desarrollo humano. Tal perspectiva tiene un gran significado.

$\mathrm{Al}$ iniciar este artículo, se señaló que la medición del sentido de compromiso se requería para considerarla en la planificación de la $\mathrm{GH}$ y en su control de gestión estratégica. Para ilustrar esta afirmación, se presenta el Cuadro de Mando Integral ([CMI], Figura 1) que corresponde al concebido por Robert Kaplan y David Norton (Kaplan $\&$ Norton, 2004), en el cual se consideraron intangibles como el referido al Índice de sentido de compromiso (Isc), previamente tomado en cuenta para la planificación en busca de su incremento (Norton, 2001). Aparecen ahí enumerados los indicadores intangibles (4, 5 y 6 ), evidenciándose los nexos causales o hipótesis entre los diferentes objetivos estratégicos planteados. Igualmente, se muestra un fragmento de la tabla donde se desglosan los objetivos planificados con sus indicadores (Tabla 8).

En el accionar de la GH en la empresa, derivado de su planificación estratégica, es imprescindible la consideración del indicador de sentido de compromiso (en uno o más de esos indicadores con validez concurrente), así como con la concepción del control de gestión estratégica, de manera que se mantenga la dirección y control de tan relevante indicador del crecimiento económico y del desarrollo humano. 
TABLA 8

Objetivos, indicadores y expresiones de cálculo de una empresa

\begin{tabular}{|c|c|c|c|c|c|c|}
\hline \multirow[t]{2}{*}{ OBJETIVOS hasta 2015} & \multirow[t]{2}{*}{ INDICADOR } & \multirow{2}{*}{$\begin{array}{l}\text { EXPRESIÓN } \\
\text { DE CÁLCULO }\end{array}$} & \multirow{2}{*}{$\begin{array}{l}\text { VALOR DE } \\
\text { REFERENCIA }\end{array}$} & \multicolumn{3}{|c|}{$\begin{array}{c}\text { METAS } \\
\text { ANU ALES }\end{array}$} \\
\hline & & & & 2013 & 2014 & 2015 \\
\hline & & & & & & \\
\hline & & & & & & $\approx$ \\
\hline $\begin{array}{l}\text { 4. Garantizar un } \\
\text { aumento de liderazgo } \\
\text { en el conjunto de jef es } \\
\text { de áreas de la } \\
\text { empresa. }\end{array}$ & $\begin{array}{l}\text { Coeficiente } \\
\text { Sociomét rico } \\
\text { del dirigente } \\
\text { (Csd) }\end{array}$ & $\operatorname{Cs} d=\sum e+{ }^{\prime} / N$ & 0.75 & 0.45 & 0.65 & 0.75 \\
\hline $\begin{array}{l}\text { 5. Aumentar el sentido } \\
\text { compromiso de los } \\
\text { empleados con la } \\
\text { empresa. }\end{array}$ & $\begin{array}{l}\text { Índice de } \\
\text { sentido de } \\
\text { compromiso } \\
\text { (Isc) }\end{array}$ & $\begin{array}{l}\Sigma \mathrm{a} c=\frac{\Sigma \mathrm{b}-\Sigma \mathrm{d}}{2}-\Sigma \mathrm{e} \\
-\frac{\mathrm{N}}{\mathrm{N}}--\end{array}$ & 2.75 & 1.35 & 2.05 & 2.75 \\
\hline $\begin{array}{l}\text { 6. Lograr que el } 100 \% \\
\text { de los trabajadores } \\
\text { alcance la idoneidad } \\
\text { acorde a los perfiles de } \\
\text { competencias de los } \\
\text { cargos que ocupan, en } \\
\text { aras de la la excelencia } \\
\text { en el servicio. }\end{array}$ & $\begin{array}{l}\text { Coeficiente } \\
\text { de idoneidad } \\
\text { demostrada. }\end{array}$ & $\begin{array}{l}\text { Cantidad de } \\
\text { trabajadores que son } \\
\text { evaluados como } \\
\text { idóneos / Cantidad } \\
\text { total de trabajadores. }\end{array}$ & $100 \%$ & $70 \%$ & $80 \%$ & $100 \%$ \\
\hline
\end{tabular}

Fuente: elaboración propia.

\section{Referencias}

Bakker, A., \& Schaufeli, W. (2008). Positive organizational behaviour: Engaged employees in flourishing organizations. Journal of Organizational Behaviour, 29, 147-154. http://dx.doi.org/10.1002/job.515

The Boston Consulting Group [BCG] \& World Federation of Personnel Management Associations. [FPMA] (2010). Creating people advantage 2010. How companies can adapt their HR practices for volatile times. Boston: Autores.

Beer, M. (2009). High commitment high performance: How to build a resilient organization for sustained advantage. Nueva York: Jossey-Bass.

Beer, M., Spector, B., Lawrence, P., Mills, Q., \& Walton, R. (1985). Human resource management. A general manager's perspectiva. Text and Cases. Nueva York: Free Press Macmillan.

Boxall, P., \& Macky, K. (2009). Research and theory in high: Performance work systems: Progressing the high-involvement stream. Human Resource
Management Journal, 19(1), 3-23. http://dx.doi. org/10.1111/j.1748-8583.2008.00082.x

Cherubin, G. (2011). Perceived organizational support and engagement (Tesis de maestría). Recuperado de http://media.proquest.com/media/pq/classic/ doc/2662547571/fmt/ai/rep/NPDF?_s=YGWRC XHR48CKrOs5GFruePPpgSE\%3D

Chih-Hsun, Ch., \& Hui, L. (2010). Strategic Human Resource Management in Service Context: Taking care of business by taking care of employees and customers. Personnel Psychology, 63(1), 153-196. http:/dx.doi.org/10.1111/j.1744-6570.2009.01165.x

Cuesta, A. (2010). Gestión del talento humano y del conocimiento. Bogotá: ECOE Ediciones.

Cuesta, A. (2011). Metodología de gestión por competencias asumiendo la norma cubana sobre gestión de capital humano. Revista Brasileira de Gestão de Negócios, 13(40), 300-311.

Cuesta, A. (2012). Modelo integrado de gestión humana y del conocimiento: una tecnología de aplicación. Revista Venezolana de Gerencia, 17(57), 84-96. 
Cuesta, A., \& Valencia, M. (2010). La productividad del trabajo del trabajador del conocimiento. Revista de Gestión de la Universidad de São Paulo, 17(4), 421-436.

Drucker, P. (1999). Knowledge-worker productivity: The biggest challenge. California Management Review, 41(2), 79-94.

Eisenberger, R., Stinglhamer, F., Van Denberghe, Ch., Sucharski, I., \& Rhoades, L. (2002). Perceived supervisor support: Contributions to perceived organizational support and employee retention. Journal of Applied Psychology, 87(3), 565-573. http:// dx.doi.org/10.1037//0021-9010.87.3.565

Gallup Consulting. (2010). Employee engagement. What's your engagement ratio? Washington, DC: Autor.

Harter, J., Schmidt, F., Killham, E., \& Aspulud, J. (2006). [Q.sup.12] meta-analysis. Princeton: The Gallup Organization.

Hernández, R., Fernández-Collado, C., \& Baptista, P. (2006). Metodología de la investigación (4. ${ }^{a}$ ed.). México: McGraw-Hill.

Kaplan, R., \& Norton, D. (2004). Strategy maps: Converting intangible assets into tangible outcomes. Boston: Harvard Business School Press.

Montoya, J. N., \& Montoya, J. D. (2012). Grupo Semco y las prácticas de recursos humanos de alto compromiso: una ventaja competitiva. Innovar, 22(46), 21-38.

Morales, A. (2006). Contribución para un modelo cubano de gestión integrada de recursos humanos (Tesis doctoral). Instituto Superior Politécnico José Antonio Echeverría - Cujae, La Habana, Cuba.

Norton, D. (2001). Medir a criação de valor: uma tarefa possível. HSM Management, 4(24), 88-94.

Oficina Nacional de Normalización. (2007). Norma Cubana NC 3001: 2007: Sistema de gestión integrada de capital humano. Requisitos. La Habana: Autor. Recuperado de www.nc.cubaindustria.cu

Peralta, M. C., Santofimio, A. M., \& Segura, V. (2007). El compromiso laboral: discursos en la organización. Revista Psicología desde el Caribe, 19, 81-109.

Picart, A. G. (2012). TRAYCO, pionera en la certificación por la NC 3001: 2007 del sistema de gestión integrada de capital humano. En D. Salazar (Coord.), La red de capital humano (pp.139-167). La Habana: Academia.

Programa de las Naciones Unidas para el Desarrollo. (2010). Informe sobre Desarrollo Humano 2010. La verdadera riqueza de las naciones: caminos al desarrollo humano. Madrid: Mundi-Prensa. Recuperado de http://hdr.undp.org/sites/default/files/ hdr_2010_es__complete_reprint.pdf

Raufflet, E., Lozano, F., Barrera, E., \& García, C. (2012). Responsabilidad social empresarial. México: Pearson Educación.

Ríos, M., Téllez, M., \& Ferrer, J. (2010). El empowerment como predictor del compromiso organizacional en las Pymes. Contaduría y Administración, 231, 103-125. Recuperado de http://www.redalyc.org/ pdf/395/39512458006.pdf

Rhoades, L., \& Eisenberger, R. (2002). Perceived organizational support: A review of the literature. Journal of Applied Psychology, 87(4), 698-714.

Schaufeli, W., Bakker, A., \& Van Rhenen, P. (2009). How changes in job demands and resources predict burnout, work engagement, and sickness absenteeism. Journal of Organizational Behavior, 30, 893-917. http://dx.doi.org/10.1002/job.595

Siegel, S. (1974). Estadística no paramétrica aplicada a las ciencias de la conducta. México: Trillas. 
